\title{
A virtual piano-playing environment for rehabilitation based upon ultrasound imaging
}

\author{
Claudio Castellini, Katharina Hertkorn, Mikel Sagardia, David Sierra González and Markus Nowak
}

\begin{abstract}
In this paper we evaluate ultrasound imaging as a human-machine interface in the context of rehabilitation. Ultrasound imaging can be used to estimate finger forces in real-time with a short and easy calibration procedure. Forces are individually predicted using a transducer fixed on the forearm, which leaves the hand completely free to operate. In this application, a standard ultrasound machine is connected to a virtual-reality environment in which a human operator can play a dynamic harmonium over two octaves, using either finger (including the thumb). The interaction in the virtual environment is managed via a fast collision detection algorithm and a physics engine.

Ten human subjects have been engaged in two games of increasing difficulty. Our experimental results, both objective and subjective, clearly show that both tasks could be accomplished to the required degree of precision and that the subjects underwent a typical learning curve. The learning happened uniformly, irrespective of the required finger, force or note. Such a system could be made portable, and has potential applications as rehabilitation device for amputees and muscle impaired, even at home.
\end{abstract}

\section{INTRODUCTION}

Standard ultrasound imaging as employed in hospitals (also called medical ultrasonography, US imaging from now on) is a non-invasive technique to visualise structures inside the human body [9] exploiting the principle of wave reflection. Piezoelectric transducers are used to generate a focused wave of ultrasound which penetrates the body part of interest; partial reflection of the wave at the interfaces between tissues with different acoustic impedance is converted to a greyscale $2 \mathrm{D}$ image (in the so-called B-mode). High values of grey denote tissue interfaces. US imaging has no known side effects [36] and is routinely used in hospitals. It can be used in, e.g., recognition of skin cancer [18], tumor segmentation [37], and anatomical landmarks detection in the foetus [27].

Recently, the usage of this technique as a human-machine interface has been employed to visualise residual lower limbs and to assess the ergonomy of lower-limb prostheses [26], [11]. As a human-machine interface for control of upperlimb prosthesis, ultrasound imaging has been explored by Zheng and others [38], [8], [17] and Castellini and others [5], [6], revealing that it can actually be used to reconstruct the hand and wrist configuration to a remarkable degree of precision. Sikdar et al. [34] have demonstrated a system able to classify finger motions, and predict finger motion velocities, based upon k-nearest-neighbours. In particular, a very fast and realistic calibration procedure has been

The authors are with the Robotics and Mechatronics Center, DLR (German Aerospace Center), 82234 Oberpfaffenhofen, Germany. email claudio.castellini@dlr.de

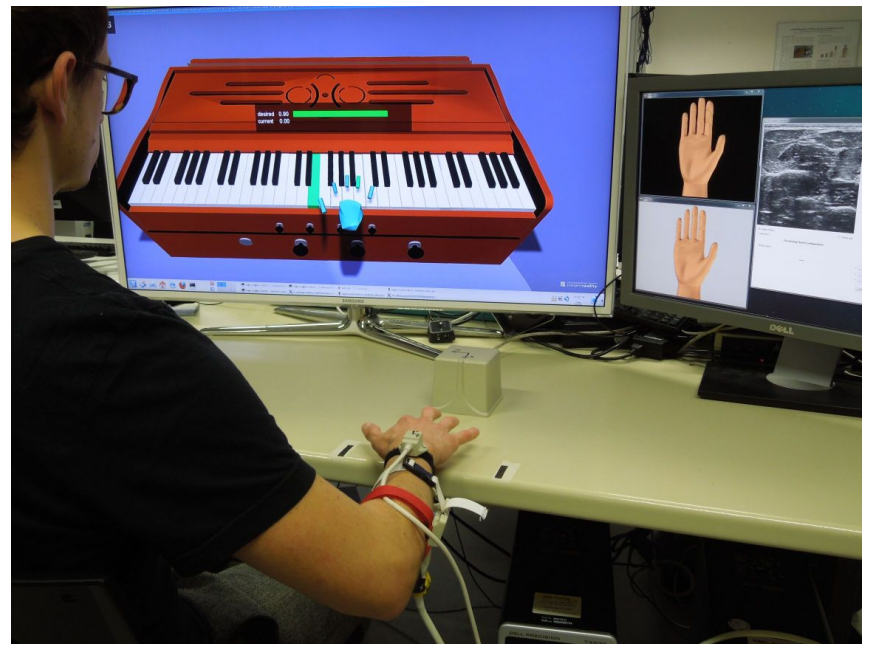

Fig. 1. The virtual-harmonium setup has three main components: an ultrasound machine capturing images of the forearm, a magnetic tracker to detect the hand movement and a realistic virtual reality environment.

developed in previous work to estimate finger forces of a human hand by employing forearm US images [33]. The system so obtained runs at $30 \mathrm{~Hz}$ and can work incrementally, meaning that the calibration can be updated at will whenever required without the need of retraining.

As a human-machine interface for virtual reality, US imaging enjoys a number of advantages with respect to standard approaches to human-machine interfaces that are specifically designed for the hand. They traditionally measure the hand kinematic configuration rather than forces and torques: instrumented gloves [10], marker-based finger tracking [1] and markerless optical tracking (Leap motion, Kinect, [23], [19]). In some cases haptic feedback is provided, improving the feeling of immersion [31] and the performance [15], [22]. Although many haptic devices and exoskeletons exist [12], [32], dexterous finger feedback remains an open topic. In contrast, US imaging can detect single finger forces as well as positions to a remarkable degree of precision [6], [33] and, since the only device needed in the online functioning is the ultrasound transducer on the forearm, it leaves the hand completely free to operate without any mechanical hampering.

This leads naturally to one main application: to reconstruct the desired finger positions and/or forces of an amputee using the US images of the stump. It is well-known [4], [35] that a remarkable amount of residual muscular activity is present in the stump even decades after the operation; 


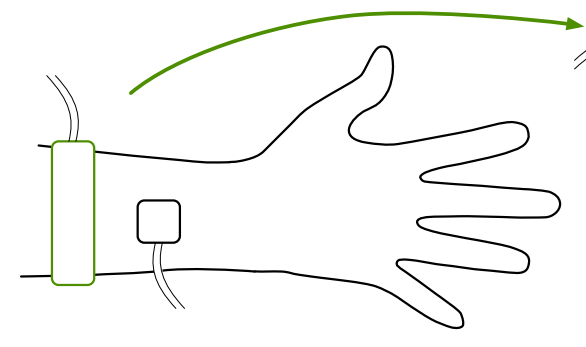

a

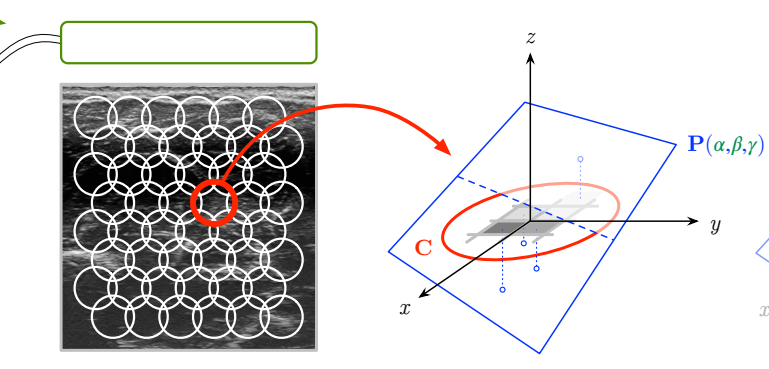

b

c

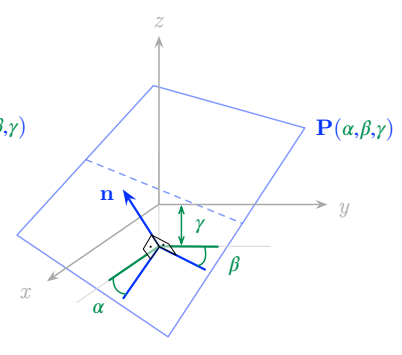

d

Fig. 2. (a) Ultrasound images of the human forearm are gathered using a standard ultrasound machine (a schematic right hand is shown); (b) Example ROIs identified on each image; (c) The grey values of each region are approximated along the $x$ and $y$ directions; (d) For each ROI $i$, three coefficients $\alpha_{i}, \beta_{i}$, and $\gamma_{i}$ are evaluated. These coefficients are the features used to estimate the finger forces. See [33].

muscular activity corresponds then to muscle/tendon/bone internal displacement and deformation, which US imaging is naturally suited to detect. Moreover, consolidated work on phantom-limb pain [28], [7] shows that mirror therapy, that is, the illusion of seeing one's missing limb in motion, is effective in reducing the pain. From this we claim that the usage of US imaging to control one's phantom/impaired limb in a scenario suitable for the general public could constitute a valid therapy for neuropathic pain.

In this paper, we demonstrate a virtual-reality system in which finger forces predicted using US imaging (coupled with a motion tracker to detect the hand motion) are used to play a dynamic keyboard musical instrument (specifically a harmonium, loaded with Hammond Organ original samples). This system is instrumental to prove that US imaging enables an extremely fine control over position of the hand and force of the fingers with minimal sensor hampering, providing a good feeling of immersion and an exciting experience. It also enables multimodal VR interaction (sound, visuals, haptics).

In order to validate the approach, we performed a psychophysical experiment involving several non-expert human subjects engaged in two harmonium-playing exercises of increasing difficulty. The results of this experiment confirm that the subjects could smoothly use this novel interface to play the instrument to the required degree of precision, meaning using each finger, ranging over two full octaves, and with different required forces - playing the full extension of musical dynamics from piano to forte. Both objective and subjective measures of performance are used and confirm that the subjects uniformly learned to use the system as the exercises progressed, reached a uniform standard of performance, and found the test engaging and smooth.

The movie provided as supplemental material to this paper shows the system as a whole, its components, and parts of the experiment.

\section{SySTEM DESCRIPTION}

The system consists of three main components: $(i)$ a standard ultrasound machine, (ii) a standard magnetic tracking device (Flock of Birds by Ascension Technologies ${ }^{1}$ ), and (iii) a virtual reality environment, as shown in Figure 1.

\footnotetext{
${ }^{1}$ www.ascension-tech.com
}

The probe of the ultrasound machine captures the images of the forearm; based on these images, the finger forces are estimated while the magnetic tracker detects the hand movement. Both the predicted forces and the hand movement are used in the virtual reality environment to play a dynamic keyboard instrument. The virtual reality simulation consists of a very fast collision detection algorithm [29] in combination with the physics engine Bullet [25]. The virtual reality also provides sound feedback with varying volume depending on the measured finger forces. The simulation is displayed on a LCD screen in 2D using the visualisation tool InstantPlayer [3].

\section{A. Ultrasound User Interface}

We hereby employ a variant of the system described in [33] — refer to this paper for a full description. Live ultrasound images of the human forearm are gathered using a standard ultrasound machine (General Electric Logiq-e $e^{2}$ ) and a 12L-RS linear transducer fixed to the forearm using a custom-built plastic cradle and a velcro strap (Figure 2a); images are captured at a rate of $30 \mathrm{~Hz}$ using a standard framegrabber. (For the details about the settings of the ultrasound machine, the reader is referred to [33].) The probe and cable weighs about 250 grams, which does not hinder the movement of the subject.

On each image, a uniform grid of regions of interest (ROIs) is identified, enabling a thorough although compact representation of the deformations induced on each image by the application of finger forces (see an example in Figure 2-b). In our case, 181 ROIs were identified, as in the above cited paper. A local spatial approximation of the grey values of the pixels in each ROI $i$ was evaluated using linear regression along the $x$ and $y$ planes, resulting in three coefficients $\alpha_{i}, \beta_{i}$, and $\gamma_{i}$ (Figure 2-c,d); the dimension of each sample is therefore $181 \cdot 3=543$.

As it was first proved in [5], there is a linear relationship between these features and the finger forces; therefore we used Ridge Regression [14] to obtain, for each finger force $f_{j} \in \mathbb{R}, j=1, \ldots, 5$, a linear predictor $f_{j}=\mathbf{w}_{j}^{T} \mathbf{v}$, where $\mathbf{v} \in \mathbb{R}^{543}$ are the features extracted from the US images. The

${ }^{2}$ www.gehealthcare.com 
regression weights are given by $\mathbf{w}_{j}=\left(\mathbf{X}^{T} \mathbf{X}+\lambda I\right)^{-1} \mathbf{X}^{T} \mathbf{y}_{j}$, where $\mathbf{X} \in \mathbb{R}^{n \times 543}$ and $\mathbf{y}_{j} \in \mathbb{R}^{n}$ accumulate all $n$ (sample,target) pairs used for training. The regularisiation coefficient $\lambda \in \mathbb{R}$ is uniformly set at the standard value of 1 in this case, and $I$ is the identity matrix. This approach has the great advantage of being bounded in space and time (i.e., it does not depend on the number of training samples but only on their dimension, in our case 543) and of being exact, therefore requiring no costly optimisation algorithm.

The Sherman-Morrison formula [13] is used to incrementally update $\mathbf{w}_{j}$, enabling the system to incorporate new knowledge at the subject's will, for instance if the calibration is deemed inaccurate for some position of the hand and forearm. The update requires no matrix inversion and is quadratic in the dimension of the input space leading to an update time of a few milliseconds. This enables retraining onthe-fly and de facto blurring the ominous distinction between a training phase and a prediction phase.

The position of the operator's hand is estimated using the magnetic tracker, whose sensor is placed on the subject's forearm, as shown in Figure 1 and 2-a. Finger forces and hand positions are streamed over UDP to the machine running the VR environment.

\section{B. Virtual Reality Environment}

The VR environment enables visual and sound feedback using fast collision detection and movement generation. We use the visualisation tool InstantPlayer [3] to visualise the environment and a standard sound player that is controlled by the predicted finger forces $f_{j}$ for the audio feedback. The collision detection is based on the Voxmap-Pointshell Algorithm [21], [29], [2], suited for haptic rendering since it computes collision forces within $1 \mathrm{~ms}$. The physics engine Bullet [25] is used to solve the motion equations. The combination of Bullet with the collision detection framework enables realistic interaction with several complex virtual objects at haptic update rates.

To detect the collisions, two types of data structures are generated offline for each colliding object-pair: voxmaps and pointshells (see Figure 3-a). Voxmaps are 3D grids that contain distance fields: each voxel stores a discrete distance value $v \in Z$ to the surface. Hence, voxels on the surface layer - the set of voxels that touch the surface of the object - have $v=0$, voxels in the $n^{\text {th }}$ inner layer have $v=n$, and voxels in the $n^{\text {th }}$ outer layer have $v=-n$. The scalar voxmap function $V(P)$ yields the penetration of a point $P$ in the voxmap. A pointshell is a set of points uniformly distributed on the surface of the object; each point $P_{i}$ has additionally an inwards pointing normal vector $\mathbf{n}_{i}$. Thus, the pointshell can be described as $\mathbf{P}=\left\{\left(P_{i}, \mathbf{n}_{i}\right)\right\}$. In this work, we use the fast and accurate data structure generator presented by [30].

During collision detection (see Figure 3-a,b), the normal vectors $\mathbf{n}_{i}$ of colliding points $P_{i}$ - those which are in voxels with $v \geq 0$ - are summed, after being weighted by their penetration in the voxmap $V\left(P_{i}\right)$, yielding the total collision force $\mathbf{F}_{\text {tot }}$. Torques $\mathbf{T}_{i}$ generated by colliding points are the cross product between forces $\mathbf{F}_{i}$ and point coordinates $P_{i}$, all

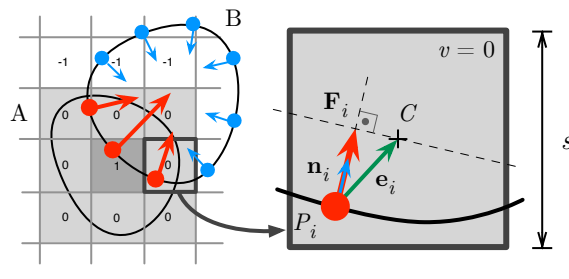

a

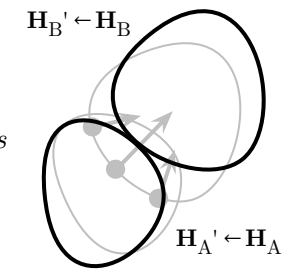

C
Fig. 3. (a) Voxelized (A) and point-sampled (B) colliding objects; (b) Single-point force $\left(\mathbf{F}_{i}\right)$ computation according to the classic VoxmapPointshell Algorithm; (c) Visualized objects are moved according to Newton-Euler motion equations or Gauss's least-constraint principle, to avoid interpenetration.

magnitudes expressed in the pointshell frame, with its origin in the center of mass. The torques $\mathbf{T}_{i}$ are then summed to compute the total torque $\mathbf{T}_{\text {tot }}$. This process is summarized in (1) and (2):

$$
\begin{gathered}
\mathbf{F}_{i}=V\left(\mathbf{x}=P_{i}\right) \cdot \mathbf{n}_{i} \rightarrow \mathbf{F}_{\text {tot }}=\sum_{\forall i \mid V\left(P_{i}\right) \geq 0} \mathbf{F}_{i} \\
\mathbf{T}_{i}=P_{i} \times \mathbf{F}_{i} \rightarrow \mathbf{T}_{\text {tot }}=\sum_{\forall i \mid V\left(P_{i}\right) \geq 0} \mathbf{T}_{i}
\end{gathered}
$$

with

$$
V\left(\mathbf{x}=P_{i}\right)=\max \{\underbrace{\left\langle\mathbf{n}_{i}, \mathbf{e}_{i}\right\rangle}_{\text {(local) }}+\underbrace{y \cdot s}_{\text {(global) }}, 0\} .
$$

The voxmap penetration function $V(P)$ has two components: global and local penetrations. The force/penetration/contact information is used to compute the appropriate (constrained) movement of the objects in the scene, yielding the homogeneous transformation matrix of each dynamic object corresponding to the next time step $\left(\mathbf{H}_{A}^{\prime}\right.$ in Figure 3-c). Since this haptic rendering algorithm is penalty-based and we do not apply any kinematic restriction to the real hand movement, Gauss's least-constraint principle is used to avoid finger-key overlap, similarly to the GodObject method [24], [16]. Using this method, we correct the homogeneous transformation matrix of each interaction object (finger phalanges), defined as $\mathbf{H}_{B}^{\prime}$ in Figure 3-c.

The virtual scenario used for the user study consists of a harmonium and a symbolic hand in which five cylindrical structures denote the fingertips (see Figure 1 again as well as the movie provided as supplemental material). The fingertip movements are controlled using force control: $q_{j}=K \cdot f_{j}$. The finger flexion angle $q_{j}$ is evaluated using the estimated finger force $f_{j}$ and a fixed stiffness $K$. The translational motion of the virtual hand is enforced directly using the magnetic tracking. We used a virtual model of a harmonium loaded with Hammond Organ samples. The contact forces between fingers and keys were used to set the the volume of the corresponding notes in a preliminary round of tests. 
TABLE I

THE STATEMENTS ADMINISTERED TO THE SUBJECTS.

\begin{tabular}{|c|c|}
\hline \multicolumn{2}{|c|}{ Questionnaires 1 and 2: general behaviour (after each game) } \\
\hline Q1 & $\begin{array}{l}\text { The behaviour of the virtual hand fully matched my expecta- } \\
\text { tions. }\end{array}$ \\
\hline Q2 & $\begin{array}{l}\text { The interaction with the virtual hand in the remote environ- } \\
\text { ment was natural. }\end{array}$ \\
\hline Q3 & It was tiresome to work with the virtual hand. \\
\hline Q4 & I had the feeling that the virtual hand was my own hand. \\
\hline Q5 & The task was easy. \\
\hline Q6 & My performance improved along with time. \\
\hline Q7 & It was easy to control the single fingers. \\
\hline Q8 & It was easy to press with the required force. \\
\hline Q9 & $\begin{array}{l}\text { I had the impression that there was a delay between the } \\
\text { movement of my hand and the virtual one. }\end{array}$ \\
\hline Q10 & $\begin{array}{l}\text { I had the impression that my hand and the virtual one did not } \\
\text { match. }\end{array}$ \\
\hline \multicolumn{2}{|c|}{ Questionnaire 3: fingers (at the end) } \\
\hline Q1-5 & $\begin{array}{l}\text { (for each finger) It was intuitive to control this finger (pinkie, } \\
\text { ring, middle, index and thumb). }\end{array}$ \\
\hline Q6-10 & $\begin{array}{l}\text { (for each finger) This finger moved the way I wanted (pinkie, } \\
\text { ring, middle, index and thumb). }\end{array}$ \\
\hline
\end{tabular}

\section{USER STUDY}

A user study including 10 subjects (age 26.1 \pm 3yrs, 1 woman and 9 men, all right-handed) was designed in order to prove the usability of the system. Before the experiment, each subject received a thorough oral and written description of the experiment and signed an informed consent form. Experiments with ultrasound imaging involving human subjects were previously approved by the Ethical Committee of our Institution.

\section{A. Experiment Protocol}

Each subject sat on a fixed chair (see Figure 1) in front of a table on which a 46" monitor would display the virtual reality environment. The US transducer was attached firmly to the ventral side of the subject's right forearm midway between the elbow and the wrist after measuring the length of his/her forearm. The magnetic tracking sensor was fixed on a velcro strap wrapped around the forearm slightly down the forearm with respect to the attachment of the US transducer. The subject was then instructed to hold the hand on the table at a height of a few centimeters, in order to press with the fingers on the table as if hitting the keys of an imaginary keyboard. Two pieces of tape on the table would denote the limits of the virtual keyboard, that is, the limits of the required motion range of the hand.

The subject was then instructed to follow a visual stimulus on another monitor, a 3D hand model alone, which would represent, in turn, the intended flexions of the five fingers; this was done once for each finger at the two extremes of the motion range. Soon after, a similar hand model would display the finger forces estimated in both positions by the system. If necessary, retraining was enforced in order to correct the prediction. This procedure ensured that the prediction was good at both extremes. (The procedure closely follows that outlined in [33].) This procedure entails no usage of force sensors - the ground truth for training is obtained by using the values of the visual stimuli.

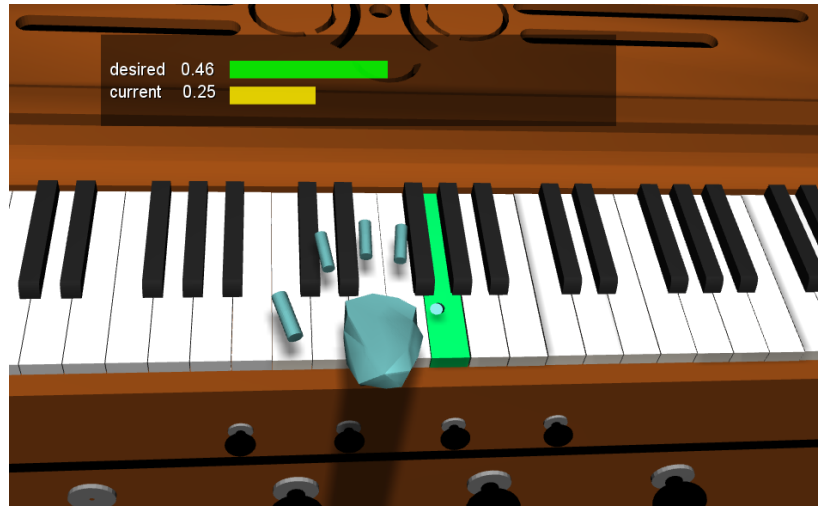

(a)

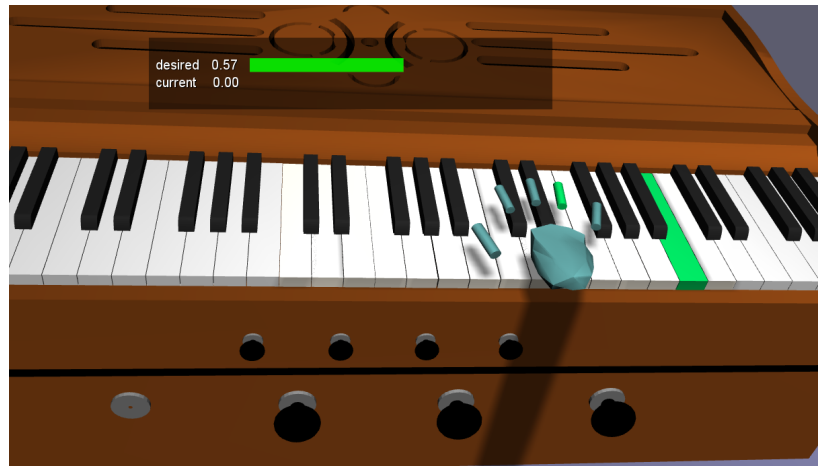

(b)

Fig. 4. (a) Game 1: The symbolic hand interacts with five keys of the harmonium (C-D-E-F-G); hand movement is restricted to the direction parallel to each key, so that each finger can reach only one key. (b) Game 2: The symbolic hand interacts with two octaves of the harmonium (C-C-C). The required finger/key combination is highlighted in green.

Two games were administered to each subject in increasing order of difficulty. In the first game (Figure 4(a)), the virtual hand was positioned so that its five fingers would lie exactly on top of the first five notes of the keyboard (C-DE-F-G); motion parallel to the keys was allowed to enable the thumb reach the $\mathrm{C}$ key. A random sequence of required notes $(\mathrm{C}$ to $\mathrm{G})$ and forces (ranging from 0 to 1 in arbitrary units $u$ ) was established and the game would then start: the key corresponding to the required note would be highlighted in green and a coloured bar just above the keyboard would show the required amount of force; the volume of the played note was set in real-time according to the required force, in order to give the subject an auditory cue as well as a visual one. (A required note/finger/force will be from now called an "attempt".) Each attempt was considered successful once the subject could hold the required note at the required volume using the required finger for at least 650 milliseconds; a tolerance range of \pm 0.2 arbitrary units was allowed for the force. Immediately after, the next attempt would start. In total, a randomised sequence of 49 attempts was required. In order to train the ultrasound system, and to make the subject acquainted with the procedure, a preliminary similar exercise was administered, in which forces were set uniformly at a value of 1 ; this exercise will be referred to as to the 
calibration phase for game 1 . The second game was identical to the first but this time the virtual hand could move across the keyboard enabling the subject to play any note on the two octaves (C-C-C) (Figure 4(b)); additionally, a particular finger, highlighted in green as well, was needed to hit the required key. In total, 74 note/key/force attempts were requested. In this case too, a calibration phase preceded the game. The calibration phases lasted on average 4'10" for the first game and 5'35" for the second, needing on average 5 single-finger training repetitions.

\section{B. Data Collection and Analysis}

a) Objective Data: During both games, the forces exerted by the subject, as estimated by the US system finger by finger, as well as the virtual hand position, were recorded; at the same time, for each step of the two games, the physical interaction engine would record the required key/finger/force combiantion, and the exact time at which the key was successfully pressed. For each attempt this defines the reaction time (RT) as the time difference between two attempts. Out of the RTs for each game we only considered the lower $85 \%$ percentile in order to avoid outliers; besides that, a few attempts (less than $1 \%$ of the total) were eliminated since the subject could not make it within a reasonable amount of time.

b) Subjective Data: After the first and second game, and at the end of the experiment, questionnaires were administered to each subject. Each statement in the questionnaires was to be judged with a Likert linear scale value [20] ranging from 1 (complete disagreement) to 7 (complete agreement). The statements regarded the easiness of control of the virtual hand and single fingers, both as far as the required position and force were concerned; moreover, we asked the subjects how tiresome each game was and if they could clearly notice whether they would get better as the game progressed.

Questionnaires 1 and 2 (identical copies) were administered in turn after the first and second game, in order to check whether the subjective judgment would change as the difficulty of the exercise increased; these two questionnaires dealt with the general behaviour of the system with respect to hand control, feeling of immersion, learning, etc. The third questionnaire evaluated the perceived feeling of control for each single finger. Table I shows the statements on the questionnaires.

\section{RESUlTS}

\section{A. Reaction times and learning trend}

The RTs obtained during both games are displayed for a typical subject in Figure 5-a, and for all subjects in Figure 5-b. Consider panel (a): the RTs for the first game are $3.35 \pm$ $1.03 \mathrm{~s}$ (mean value plus/minus one standard deviation), while for the second game they are $5.40 \pm 1.50 \mathrm{~s}$. The mean values are stastically different from each other (Student's two-tailed t-test p-value smaller than 0.01) as it is to be reasonably expected. An exponential fit of the two datasets $\left(e^{\alpha x+\beta}\right)$ yields $\alpha=-0.0071$ for the first game and $\alpha=-0.0027$ for the second, confirming the existence of a learning trend.
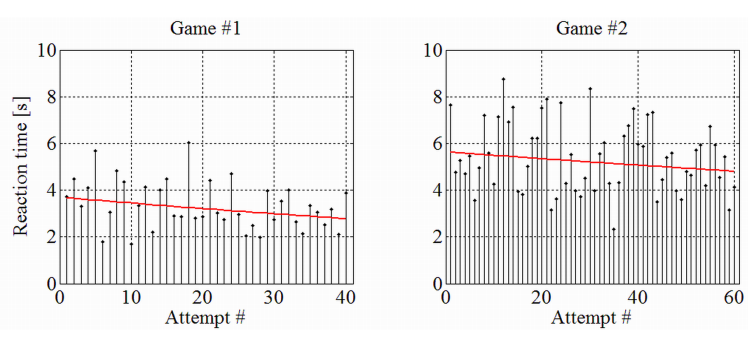

(a)

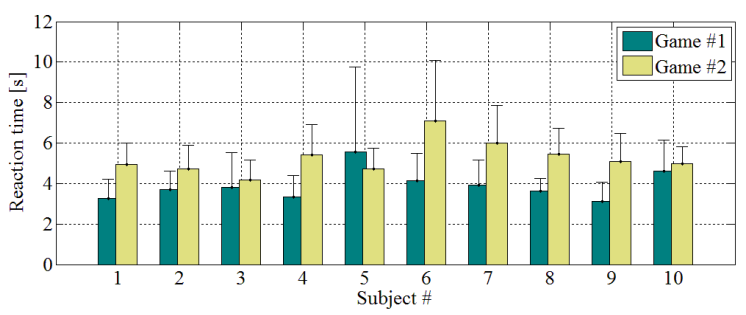

(b)

Fig. 5. Reaction times for each game. (a) Detail for a typical subject (red curve: exponential fit); (b) Results for all subjects and for each game.

Consider now panel (b): a similar evaluation for all subjects reveals that in general the RTs for game 1 are lower then those for game 2 (mean values $3.92 \mathrm{~s}$ and 5.25s), with a statistically significant difference for 7 subjects out of 10 (3, 5 and 10 being the exceptions). A negative $\alpha$ coefficient is present in 8 out of 10 cases for the first game, and in 7 out of 10 cases for the second game. No other significant statistical deviations are detected. This confirms that in general, the second game was harder to complete than the first and that a learning trend occurred.

\section{B. Correlation with Other Factors}

Surprisingly, weak or no correlation exists between the RTs and, in turn, the required finger (maximum Pearson correlation coefficient over all subjects 0.42 for the first game, 0.31 for the second), the required note $(0.25$ and $0.28)$, and the required force $(0.42$ and 0.31$)$. Moreover, there is weak or no correlation also between the RT and the distance between the last and the current required note $(0.40$ and 0.30), which sounds counterintuitive, since the subject's hand must physically travel from the previous position to the current one to accomplish the attempt.

These results indicate that the learning has happened uniformly, irrespective of any other parameter required of each subject.

\section{Subjective Evaluation}

Figure 6 shows the results of the subjective evaluation. Consider the left panel: first and foremost, note that there is no statistically significant difference between the values related to game 1 and game 2 (Student's two-tailed t-test pvalue larger than 0.05 for all 10 questions). This indicates that, notwithstanding the increased difficulty of game 2 with respect to game 1, no difference in the general behaviour of the system was noticed. As far as single questions are 

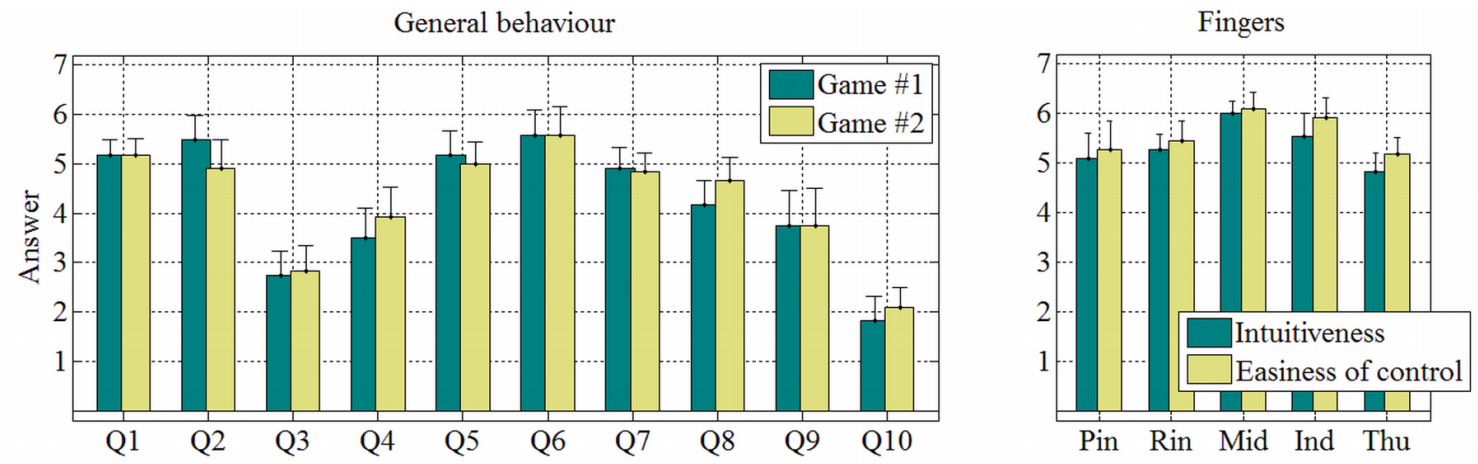

Fig. 6. Subjective evaluation of the games, for all subjects, question by question. (left) General perceived behaviour of the system, after game 1 and game 2; (right) Intuitiveness and and easiness of control at the end of the experiment. Mean values of the answers (1-7 Likert scale) plus/minus one standard error of the mean.

concerned, notice that the evaluation is high (around 5) for Q1 (coherent behaviour of the hand) and Q2 (natural interaction with the environment). The task was found to be rather not tiresome (Q3, average value around 3), and it was easy to control the system (Q5), the single fingers (Q7) and the required force (Q8). A control question, regarding the discrepancy between the subject's hand and the virtual hand received, coherently, a rather low score (Q10, average around 2). It must be noted that some delay was perceived between the movements of the real and virtual hand (Q9, average value around 4); in fact the feeling of immersion was not rated very high (Q4, average value lower than 4). Lastly, notice that a learning effect was correctly uniformly perceived (Q6, average value of about 5.5).

In the right panel, the subjective evaluation for single fingers is displayed. All fingers were on average equally perceived as intuitive and easy to control, with a slight preference for the middle and index fingers with respect to the others. No stastically significant difference between the responses for the same question in the two games was observed (Student's t-test p-value always greater than 0.05 ).

\section{COnClusion And Future Work}

In this paper we have described and practically demonstrated an application of a human-machine interface based upon medical ultrasound imaging. Ultrasound images are used in real-time to estimate finger forces of a human hand. This estimation, together with the hand position tracked with a standard magnetic tracking device, is then used to control a multifingered virtual hand playing a virtual harmonium. The virtual harmonium allows to dynamically play two full octaves with any of the five fingers. The system is tightly integrated so that a fine control over the finger forces and hand positon is enforced, enabling non-expert human subjects to play this instrument without wearing any sensors on the hand. A user study involving ten human subjects reveals that the interface is easy to use, quickly calibrated and intuitive; the integrated system provides realistic control of the virtual hand, leading to an exciting experience. A learning effect is present in the user study, confirmed both numerically and subjectively; the learning happens irrespective of the required finger to use, the required force to apply and the required note to be played.

The system hereby presented enables fine control over single finger forces using a device which is attached to the proximal section of the forearm. Given that fine residual muscular activity is left in an amputee's stump even decades after the operation [4], [35], we claim that its most interesting application in the mid-term is to employ it with disabled subjects who suffer from neuropathic pain (hand amputees with phantom-limb pain, patients of Complex Regional Pain Syndrome, etc.). In fact, playing a virtual instrument with the missing/impaired limb can be seen as an exciting experience, in which the defective sensorimotor loop is to some extent restored, actually in a deeper way than what happens in mirror therapy [28], [7], which has been proven to be effective in countering such disabling conditions.

Future work includes a study of the interaction of the different fingers in order to be able to detect multi-fingered forces. The system is as well being optimised to reduce the delay between the action of the subject and the response of the VR environment. One current limitation of the system is that it is very sensitive to wrist rotation, which limits the applicability to tasks which happen on a planar range; in order to counter this problem, we are studying an optimised training strategy, as well as the usage of image transformation techniques such as, e.g., the optical flow detection.

\section{REFERENCES}

[1] Advanced Realtime Tracking GmbH. The ART fingertracking system, www.ar-tracking.com/products/interaction-devices/fingertracking, 2013. Accessed November 1st, 2013.

[2] J. Barbič and D. James. Six-dof haptic rendering of contact between geometrically complex reduced deformable models. IEEE Transactions on Haptics, 1(1):39 -52, jan.-june 2008.

[3] J. Behr, P. Dähne, Y. Jung, and S. Webel. Beyond the web browser - X3D and immersive VR. In Proc. of the IEEE Symp. on 3D User Interfaces (3DUI), 2007.

[4] C. Castellini, A. E. Fiorilla, and G. Sandini. Multi-subject / dailylife activity EMG-based control of mechanical hands. Journal of Neuroengineering and Rehabilitation, 6(41), 2009.

[5] C. Castellini and G. Passig. Ultrasound image features of the wrist are linearly related to finger positions. In Proc. IEEE/RSJ Int. Conf. on Intelligent Robots and Systems, pages 2108-2114, 2011. 
[6] C. Castellini, G. Passig, and E. Zarka. Using ultrasound images of the forearm to predict finger positions. IEEE Transactions on Neural Systems and Rehabilitation Engineering, 20(6):788-797, 2012.

[7] B. L. Chan, R. Witt, A. P. Charrow, A. Magee, R. Howard, P. F. Pasquina, K. M. Heilman, and J. W. Tsao. Mirror therapy for phantom limb pain. New England Journal of Medicine, 357(21):2206-2207, 2007.

[8] X. Chen, Y.-P. Zheng, J. Y. Guo, and J. Shi. Sonomyography (SMG) control for powered prosthetic hand: a study with normal subjects. Ultrasound in Med. \& Biol, 36(7):1076-1088, 2010.

[9] R. S. C. Cobbold. Foundations of Biomedical Ultrasound. Biomedical Engineering. Oxford University Press, 2007.

[10] L. Dipietro, A. Sabatini, and P. Dario. A survey of glove-based systems and their applications. IEEE Transactions on Systems, Man, and Cybernetics, Part C: Applications and Reviews, 38(4):461-482, 2008.

[11] T. Douglas, S. Solomonidis, W. Sandham, and W. Spence. Ultrasound imaging in lower limb prosthetics. Neural Systems and Rehabilitation Engineering, IEEE Transactions on, 10(1):11-21, 2002.

[12] F. Gosselin, C. Andriot, J. Savall, and J. Martn. Large workspace haptic devices for human-scale interaction: A survey. In M. Ferre, editor, Haptics: Perception, Devices and Scenarios, volume 5024 of Lecture Notes in Computer Science, pages 523-528. Springer Berlin Heidelberg, 2008.

[13] W. Hager. Updating the inverse of a matrix. SIAM Review, 31(2):221239, 1989.

[14] A. E. Hoerl and R. W. Kennard. Ridge regression: Biased estimation for nonorthogonal problems. Technometrics, 12:55-67, 1970.

[15] J. Hummel, R. Wolff, J. Dodiya, A. Gerndt, and T. Kuhlen. Short paper: Towards interacting with force-sensitive thin deformable virtual objects. In Proc. Eurographics Association. Joint Virtual Reality Conference of ICAT - EGVE - EuroVR, pages 17-20, 2012.

[16] J. Jacobs, M. Stengel, and B. Fröhlich. A generalized god-object method for plausible finger-based interactions in virtual environments. In Proc. of the IEEE Symp. on 3D User Interfaces (3DUI), 2012.

[17] G. Jing-Yi, Z. Yong-Ping, L. P. J. Kenney, A. Bowen, D. Howard, and J. J. Canderle. A comparative evaluation of sonomyography, electromyography, force and wrist angle in a discrete tracking task. Ultrasound in Med. \& Biol, 37(6):884—891, 2011.

[18] T. M. Jørgensen, A. Tycho, M. Mogensen, P. Bjerring, and G. B. Jemec. Machine-learning classification of non-melanoma skin cancers from image features obtained by optical coherence tomography. Skin Research and Technology, 14(3):364-369, 2008.

[19] D. Kim, O. Hilliges, S. Izadi, A. D. Butler, J. Chen, I. Oikonomidis, and P. Olivier. Digits: freehand $3 \mathrm{~d}$ interactions anywhere using a wrist-worn gloveless sensor. In Proc. 25th annual ACM sympo. on User interface software and technology, pages 167-176, 2012.

[20] R. Likert. A technique for the measurement of attitudes. Archives of Psychology, 22(140):1-55, 1932.

[21] W. A. McNeely, K. D. Puterbaugh, and J. J. Troy. Voxel-based 6-dof haptic rendering improvements. Haptics-e: The Electronic Journal of Haptics Research, 3, 2006.

[22] M. Moehring and B. Froehlich. Effective manipulation of virtual objects within arm's reach. In Proc. of the IEEE Virtual Reality Conference (VR), pages 131-138, 2011.

[23] D. Mohr and G. Zachmann. A survey of vision-based markerless hand tracking approaches. Preprint submitted to Computer Vision and Image Understanding., 2013.

[24] M. Ortega, S. Redon, and S. Coquillart. A six degree-of-freedom god-object method for haptic display of rigid bodies with surface properties. In IEEE Transactions on Visualization and Computer Graphics, 2007.

[25] B. Physics. Bullet, http://bulletphysics.org/, 2013. accessed September 11 th, 2013.

[26] H. Ping, K. Xue, and P. Murka. 3-D imaging of residual limbs using ultrasound. Journal of Rehabilitation Research and Development, 34(3):269-278, 1997.

[27] B. Rahmatullah, A. Papageorghiou, and J. Noble. Image analysis using machine learning: Anatomical landmarks detection in fetal ultrasound images. In Computer Software and Applications Conference (COMPSAC), 2012 IEEE 36th Annual, pages 354-355, 2012.

[28] V. S. Ramachandran, D. Rogers-Ramachandran, and S. Cobb. Touching the phantom limb. Nature, 377(6549):489-490, 1995.

[29] M. Sagardia and T. Hulin. Fast and accurate distance, penetration, and collision queries using point-sphere trees and distance fields. In SIGGRAPH Posters, page 83, 2013.

[30] M. Sagardia, T. Hulin, C. Preusche, and G. Hirzinger. Improvements of the voxmap-pointshell algorithm - fast generation of haptic data structures. In Proc. 53rd Int. Wissenschaftliches Kolloquium, 2008.

[31] M. Sagardia, B. Weber, T. Hulin, C. Preusche, and G. Hirzinger. Evaluation of visual and force feedback in virtual assembly verifications. In Proc. of the IEEE Virtual Reality Conference (VR), 2012.

[32] K. Shimoga. A survey of perceptual feedback issues in dexterous telemanipulation. i. finger force feedback. In Proc. on the Virtual Reality Annual International Symposium, pages 263-270, 1993.

[33] D. Sierra González and C. Castellini. A realistic implementation of ultrasound imaging as a human-machine interface for upper-limb amputees. Frontiers in Neurorobotics, 7(17), 2013.

[34] S. Sikdar, H. Rangwala, E. B. Eastlake, I. A. Hunt, A. J. Nelson, J. Devanathan, A. Shin, and J. J. Pancrazio. Novel method for predicting dexterous individual finger movements by imaging muscle activity using a wearable ultrasonic system. Neural Systems and Rehabilitation Engineering, IEEE Transactions on, 22:69-76, 2014.

[35] F. V. Tenore, A. Ramos, A. Fahmy, S. Acharya, R. Etienne-Cummings, and N. V. Thakor. Decoding of individuated finger movements using surface electromyography. IEEE Trans. Biomed. Eng., 56(5):14271434, 2009.

[36] World Health Organisation. Training in diagnostic ultrasound: essentials, principles and standards: report of a WHO study group. WHO Technical Report Series, nr. 875. World Health Organisation, 1998.

[37] J. Zhang, K. Ma, M. Er, and V. Chong. Tumor segmentation from magnetic resonance imaging by learning via one-class support vector machine. In International Workshop on Advanced Image Technology (IWAIT '04), pages 207-211, 2004.

[38] Y. Zheng, M. Chan, J. Shi, X. Chen, and Q. Huang. Sonomyography: Monitoring morphological changes of forearm muscles in actions with the feasibility for the control of powered prosthesis. Medical Engineering \& Physics, 28:405-415, 2006. 